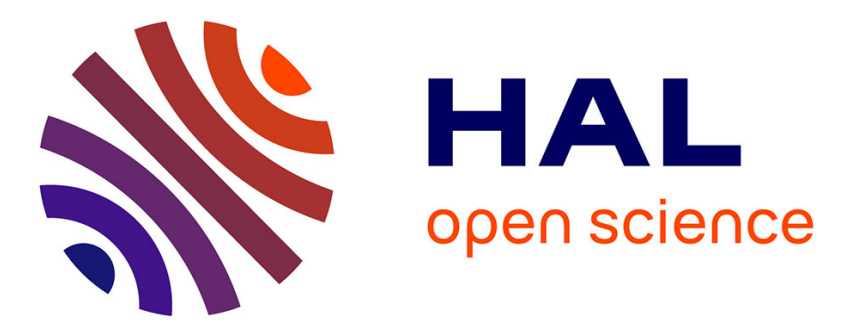

\title{
COMPARAISON DE DEUX MÉTHODES DE MESURE DE LA PUISSANCE ACOUSTIQUE PAR INTENSIMÉTRIE : POINTS FIXES ET BALAYAGE
}

P. Daniere, J. Sueur

\section{- To cite this version:}

P. Daniere, J. Sueur. COMPARAISON DE DEUX MÉTHODES DE MESURE DE LA PUISSANCE ACOUSTIQUE PAR INTENSIMÉTRIE: POINTS FIXES ET BALAYAGE. Journal de Physique Colloques, 1990, 51 (C2), pp.C2-225-C2-228. 10.1051/jphyscol:1990254 jpa-00230675

HAL Id: jpa-00230675 https://hal.science/jpa-00230675

Submitted on 1 Jan 1990

HAL is a multi-disciplinary open access archive for the deposit and dissemination of scientific research documents, whether they are published or not. The documents may come from teaching and research institutions in France or abroad, or from public or private research centers.
L'archive ouverte pluridisciplinaire HAL, est destinée au dépôt et à la diffusion de documents scientifiques de niveau recherche, publiés ou non, émanant des établissements d'enseignement et de recherche français ou étrangers, des laboratoires publics ou privés. 
COLLOQUE DE PHYSIQUE

Colloque C2, supplément au $n^{\circ} 2$, Tome 51, Février 1990

ler Congrès Français d'Acoustique 1990

COMPARAISON DE DEUX MÉTHODES DE MESURE DE LA PUISSANCE ACOUSTIQUE PAR INTENSIMETRIE : POINTS FIXES ET BALAYAGE

P. DANIERE et J. SUEUR

Institut National de Recherche et de Sécurité (I.N.R.S.), Centre de Recherche, Avenue de Bourgogne, BP. 27, F-54501 Vandoeuvre-1es-Nancy, France

Résumé - On compare ici le résultat de détermination du niveau de puissance acoustique par intensimétrie obtenu par la technique des points fixes à celui obtenu par la technique de balayage, pour différentes conditions expérimentales. Les écarts constatés sont faibles et restent inférieurs le plus souvent à $\pm 0,5 \mathrm{~dB}$.

Abstract - In this paper, the résults of sound power level measurements using a fixed points intensity measuring technique are compared to the measurements obtained when using sweeping intensity measuring technique, under differents experimental conditions. The differences are very small and in most cases stay within $\pm 0,5 \mathrm{~dB}$

\section{1 - INTRODUCTION}

La détermination de la puissance acoustique Lw des sources industrielles de bruit sur leur site d'exploitation est devenue possible grâce à la technique de l'intensimétrie acoustique. Cependant le développement de cette technique est entravé par différents obstacles. L'un d'entre eux concerne la durée de la mesure, notamment lorsque l'environnement immédiat de la source à mesurer perturbe le champ acoustique. Dans ce cas, l'obtention de la précision de "classe expertise" pour la détermination de $\mathrm{Lw}$ nécessite un grand nombre de points de mesure. II est souvent nécessaire d'utiliser plus de 50 points pour des machines de l'ordre de 1 à $2 \mathrm{~m}^{3}$. Pour ces situations rencontrées fréquemment en milieu industriel, l'intérêt suscité par l'intensimétrie est fortement réduit du fait de la durée trop longue nécessaire à l'acquisition des données.

C'est pourquoi des études ont été conduites pour développer, dans le cadre de l'intensimétrie, une technique d'acquisition plus rapide, dite de balayage, consistant à balayer avec la sonde intensimétrique une surface de mesure entourant la source sonore. La comparaison des résultats obtenus en utilisant la technique des emplacements fixes de la sonde avec ceux obtenus par la technique de balayage n'a fait l'objet jusqu'à présent que de quelques publications [1 à 6]. Elles montrent cependant que les écarts obtenus en utilisant les deux techniques sont faibles; ils ne dépassent guère $0,5 \mathrm{~dB}$ par bande de tiers ou d'octave. Ces résultats expérimentaux sont corroborés par des simulations numériques, effectuées dans des situations idéales $[7,8]$.

Pour confirmer l'intérêt de la technique de balayage et en préciser la plage d'utilisation, des mesures de puissance acoustique ont été entreprises à l'aide de machines réelles et en utilisant les deux techniques mentionnées: points fixes et balayage.

Pour ces essais, quatre machines ont été utilisées : une source sonore de référence type EDF, une dégauchisseuse, un aspirateur de copeaux, un climatiseur.

Les essais se sont déroulés d'une part dans une chambre semi-anéchoïque (champ libre sur plan réfléchissant) et d'autre part dans divers locaux de type industriel.

\section{2 - UTILISATION DELA TECHNIQUE DE BALAYAGE DE LA SONDE SURUNE SURFACE DE $1 \mathrm{~m}^{2}$.}

La surface de mesure entourant la source et située à $0,5 \mathrm{~m}$ de la surface de référence est découpée en cellules de $1 \mathrm{~m}$ par $1 \mathrm{~m}$ sur lesquelles sont effectuées les mesures. Les puissances acoustiques $L w$ de deux sources sonores (source de référence et dégauchisseuse) ont été déterminées à l'aide de deux méthodes différentes:

la technique des emplacements fixes de la sonde avec une densité de 4 points de mesure par cellule de $1 \mathrm{~m}^{2}$ référencée $\mathrm{Lw}\left(4 / \mathrm{m}^{2}\right)$ (figure 1a). 
- la seconde méthode référencée $L w(B a l)$ emploie deux techniques de balayage :

* un balayage court, effectué pour chaque cellule de $1 \mathrm{~m}^{2}$, sur une longueur de l'ordre de $2 \mathrm{~m}$ comme le montre la figure ta, Lw(Bal1).

* un balayage long effectué pour chaque cellule de $1 \mathrm{~m}^{2}$ mais sur une longueur égale à $4 \mathrm{~m}$, Lw(Bal2) (figure 1a).

Parmi les écarts de puissance acoustique $\Delta L\left(\Delta L=L w(B a l)-L w\left(4 / m^{2}\right)\right)$, obtenus en utilisant ces deux méthodes (points fixes et balayage), pour différentes conditions expérimentales, on observe que la technique du balayage long donne les résultats les plus proches de la valeur obtenue avec la technique des points fixes. En effet l'écart $\Delta L$ varie de $+0,2$ à $-0,8 \mathrm{~dB}$ en champ libre et de $+0,8$ à $-0,9 \mathrm{~dB}$ sur site. Ces résultats confirment partiellement ceux obtenus en champ libre par Tachibana [1] qui obtient des écarts de puissance acoustique $\Delta L$ presque toujours négatifs lors d'essais effectués dans des conditions expérimentales comparables aux nôtres.

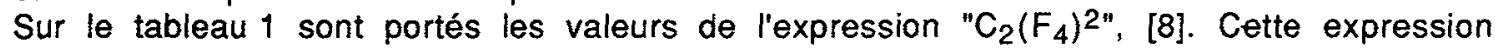
fait intervenir l'indicateur de champ " $F_{4}$ " qui caractérise l'hétérogénéité du champ acoustique et un coefficient $" \mathrm{C}_{2}$ " qui est fonction du degré de précision souhaité. $\mathrm{Ce}$ critère permet un contrôle, a posteriori, de l'adéquation du maillage choisi à la précision souhaitée. Les valeurs portées sur le tableau 1 montrent que les mesures effectuées à l'aide de la technique des points fixes, en utilisant une densité de 4 points par $\mathrm{m}^{2}$, entrent dans la "classe laboratoire" aussi bien pour les essais entrepris en champ libre que pour ceux effectués sur site.

Pour les mesures de puissance acoustique effectuées à l'aide de la technique de balayage, les indicateurs de champ n'ont pas encore été définis. Cependant, compte tenu des faibles écarts observés pour la détermination de $L w$ par ces deux méthodes, points fixes et balayage, on peut penser que la technique du balayage permet d'obtenir des valeurs de $\mathrm{Lw}$ d'une précision au moins égale à celle de la "classe expertise". Précisons que cette technique reste encore assez longue à mettre en oeuvre car elle nécessite un balayage sur chacune des cellules de $1 \mathrm{~m}^{2}$ constituant la surface de mesure entourant la source sonore.

\section{3 - UTILISATION DE LA TECHNIQUE DE BALAYAGE SUR L'ENSEMBLE DE CHACUNE DES FACES} DUPARALLELÉPIPEDE DE MESURE ENTOURANTLA SOURCE

La technique utilisée consiste à balayer avec la sonde intensimétrique chacune des faces du parallèlipipède de mesure entourant la source. Ce balayage est effectué avec une vitesse aussi régulière que possible, de l'ordre de 0,1 à $0,2 \mathrm{~m} / \mathrm{s}$, et suivant le trajet indiqué figure $1 \mathrm{~b}$.

Les puissances acoustiques $L w$ de deux sources sonores (l'aspirateur de copeaux et le climatiseur) ont été déterminées à l'aide de deux méthodes:

La première utilise la technique des emplacements fixes de la sonde avec une densité de 4 points de mesure par $\mathrm{m}^{2}$ pour le climatiseur (référencée $L w\left(4 / \mathrm{m}^{2}\right)$ ), et avec une densité de 1 point par $\mathrm{m}^{2}$ pour l'aspirateur (référencée $L w\left(1 / \mathrm{m}^{2}\right)$ ). Cette dernière densité de 1 point par $\mathrm{m}^{2}$ a été retenue pour évaluer le degré de précision de la méthode des points fixes lors de son utilisation sur le site.

- La seconde méthode emploie la technique de balayage illustrée par la figure 1b, référencée $L w(B a l)$.

Les écarts de puissance acoustique $\Delta L=L w(B a l)-L w\left(4 / m^{2}\right)$ (pour le climatiseur) et $\Delta L=L w(B a l)-L w\left(1 / m^{2}\right)$ (pour l'aspirateur), relevés en utilisant ces deux techniques dans différents environnements sont donnés sur la figure 2.

L'écart $\Delta L$ constaté, sur site, est limité à $\pm 0,5 \mathrm{~dB}$ pour l'aspirateur de copeaux (figure 2b) et compris entre $+0,2 \mathrm{~dB}$ et $+1 \mathrm{~dB}$ pour le climatiseur (figure $2 \mathrm{c}$ ). En champ libre sur plan réfléchissant et pour l'aspirateur de copeaux (figure 2a), les écarts $\Delta L$ sont semblables à ceux obtenus sur site; ils sont limités à $\pm 0,5 \mathrm{~dB}$. Ces résultats confirment ceux de Brock [2] qui constate lui aussi, lors d'essais effectués en champ libre sur plan 
réfléchissant des écarts entre les mesures effectuées par balayage et par points fixes, inférieurs à $0,7 \mathrm{~dB}$ par bande d'octave.

\section{4 - ESSAIS DE REPRODUCTIBILITÉ}

Pour préciser l'influence de l'opérateur sur la mesure de la puissance acoustique par balayage (respect de la vitesse de balayage, du trajet à parcourir), des essais ont été conduits à l'aide de trois opérateurs différents. Ils ont portés sur la même machine (l'aspirateur de copeaux), dans des conditions d'environnement identiques (sur site). Ces mesures ont été réalisées par la technique du balayage effectué sur l'ensemble de chaque face du parallèlipipède de mesure (figure 1b).

De façon analogue aux mesures précédentes, on a calculé les écarts de puissance acoustique $\Delta \mathrm{L},\left(\Delta \mathrm{L}=\mathrm{Lw}(\mathrm{Bal}, \mathrm{i})-\mathrm{Lw}\left(1 / \mathrm{m}^{2}\right)\right)$, obtenus d'une part en utilisant la technique de balayage et l'opérateur $i(L w(B a l, i))$ et d'autre part la technique des points fixes $\left(\mathrm{Lw}\left(1 / \mathrm{m}^{2}\right)\right.$. Les résultas obtenus $\Delta \mathrm{L}$ sont donnés sur la figure $2 \mathrm{~d}$. On constate une faible dispersion de ces valeurs. Elle est de l'ordre de $\pm 0,15 \mathrm{~dB}$ pour toutes les fréquences à l'exception de la bande d'octave de $125 \mathrm{~Hz}$. Pettersen et Newmann [3] donnent des résultats d'essais de répétabilité effectués sur 5 mesures et pour lesquels ils obtiennent un écart type de $0,5 \mathrm{~dB}$ par tiers d'octave. Ils présentent en outre des essais de reproductibilité effectués à l'aide de 4 opérateurs pour lesquels ils obtiennent un écart type de 0,5 dB par tiers d'octave également. Ces essais ont été effectués sur une grande machine $(20 \mathrm{~m} \times 4 \mathrm{~m} \times 2 \mathrm{~m})$; or celle utilisée pour nos mesures étant plus petite $(3 \mathrm{~m} \times 2 \mathrm{~m} \times 4 \mathrm{~m})$, ceci peut expliquer les valeurs plus faibles constatées au cours de nos essais.

\section{5 - CONCLUSION}

Les trois séries d'essais effectués confirment globalement les résultats décrits dans la bibliographie. La comparaison effectuée ici avec les résultats obtenus avec des emplacements fixes a permis de valider expérimentalement la technique de balayage de la sonde sur la surface entière de chaque face du parallèlèpipède. Elle a permis en outre d'évaluer l'écart existant entre ces deux techniques (de l'ordre de $\pm 0,5 \mathrm{~dB}$ ) et d'associer à cette technique de balayage une classe de précision qui est au moins (dans les cas traités ici) égale ou supérieure à la classe "expertise"

\section{REFERENCES BIBLIOGRAPHIQUES}

[1] TACHIBANA H., "Investigations on the sound power measurement by the sound intensity method using reference sources", ISO/TC43/SC1/WG25 and WG28 meetings documents, TORONTO, oct 1988.

[2] BROCK M., "Sound power measurements using different scanning techniques", Nordic Acoustical Meeting, 1988, proceedings pp 117-120.

[3] PETTERSEN 0., NEWMAN M., "An intensity method for determining sound power in situ", Noise Control Engineering Journal 1988, vol.31, number 2 , pp 93-100.

[4] DANIERE P., SUEUR J., "Contribution towards a measurement method of the acoustic power of machines via intensity", Inter-Noise 88, proceedings pp 1117-1120.

[5] CROCKER M., "Sound power determination from sound intensity - To scan or not to scan", Noise Control Engineering Journal 1986, vol 27, number 3, pp 67.

[6] TANDON N., "The effect of sound intensity measurement parameters on sound power determination", J.S.V. 1989, vol 132, number 3, pp 451-455.

[7] BOCKHOFF M., "Some remarks on the continuous sweeping method in sound power determination", Inter-Noise 84, proceedings pp 1173-1176.

[8] POPE J., "Evaluation of accuracy of sound power determination using space-averaged intensity measurements", Noise-Con 88, proceedings pp 459-462. 


\begin{tabular}{|c|c|c|c|c|c|c|c|c|}
\hline \multirow{2}{*}{$\begin{array}{l}\text { Source } \\
\text { sonore }\end{array}$} & \multirow{2}{*}{ Environnement } & \multirow{2}{*}{$\begin{array}{l}\text { Nb Total de } \\
\text { points de } \\
\text { mesures }\end{array}$} & \multicolumn{6}{|c|}{ Fréquences par bande d'octave } \\
\hline & & & 125 & 250 & 500 & 1000 & 2000 & 4000 \\
\hline Partie A : & lasse de précisio & "Expertise & & & & & & \\
\hline $\begin{array}{l}\text { Source de } \\
\text { référence }\end{array}$ & $\begin{array}{c}\text { champ libre } \\
\text { sur site }\end{array}$ & $\begin{array}{r}48 \\
48 \\
\end{array}$ & $\begin{array}{l}4 \\
4 \\
\end{array}$ & $\begin{array}{l}6 \\
7 \\
\end{array}$ & $\begin{array}{l}8 \\
8 \\
\end{array}$ & $\begin{array}{l}8 \\
9 \\
\end{array}$ & $\begin{array}{l}5 \\
4 \\
\end{array}$ & $\begin{array}{r}10 \\
8 \\
\end{array}$ \\
\hline Dégauchisseuse & $\begin{array}{l}\text { champ libre } \\
\text { sur site }\end{array}$ & $\begin{array}{l}188 \\
188 \\
\end{array}$ & $\begin{array}{l}7 \\
5\end{array}$ & $\begin{array}{l}15 \\
18 \\
\end{array}$ & $\begin{array}{l}18 \\
19\end{array}$ & $\begin{array}{l}14 \\
29 \\
\end{array}$ & $\begin{array}{l}33 \\
26 \\
\end{array}$ & $\begin{array}{l}29 \\
39 \\
\end{array}$ \\
\hline Partie B : & lasse de précisio & Laboratoir & & & & & & \\
\hline $\begin{array}{l}\text { Source de } \\
\text { référence }\end{array}$ & $\begin{array}{l}\text { champ libre } \\
\text { sur site }\end{array}$ & $\begin{array}{l}28 \\
48\end{array}$ & $\begin{array}{l}6 \\
7\end{array}$ & $\begin{array}{l}10 \\
12\end{array}$ & $\begin{array}{l}15 \\
14\end{array}$ & $\begin{array}{l}25 \\
28\end{array}$ & $\begin{array}{l}13 \\
13\end{array}$ & $\begin{array}{l}31 \\
25\end{array}$ \\
\hline Dégauchisseuse & $\begin{array}{l}\text { champ libre } \\
\text { sur site }\end{array}$ & $\begin{array}{l}188 \\
188\end{array}$ & $\begin{array}{r}14 \\
8\end{array}$ & $\begin{array}{l}26 \\
33\end{array}$ & $\begin{array}{l}33 \\
34 \\
\end{array}$ & $\begin{array}{l}44 \\
92 \\
\end{array}$ & $\begin{array}{r}105 \\
82\end{array}$ & $\begin{array}{r}92 \\
123 \\
\end{array}$ \\
\hline
\end{tabular}

Tableau 1 - Résultats de calculs de l'expression " $C_{2} F_{4}{ }^{2}$ " [8] obtenus pour les différentes sources et les différentes conditions expérimentales utilisées.

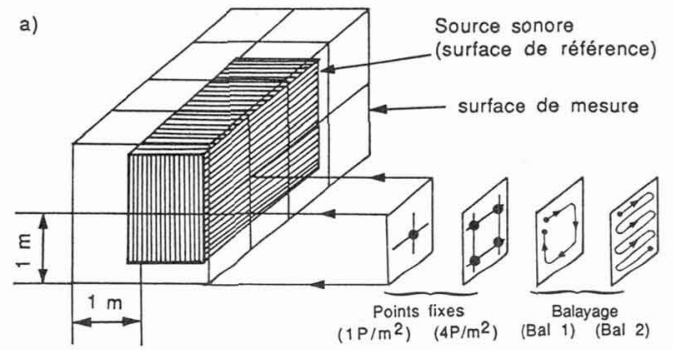

b)

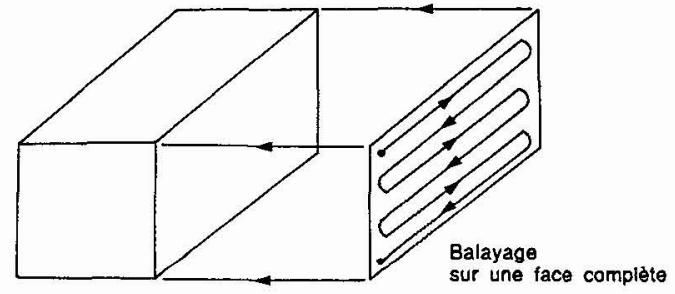

Figure 1 - 1a) Illustration des techniques de balayage utilisées lorsque la surface de mesure est décomposée en cellules de $1 \mathrm{~m}^{2}$.

1b) Exemple de trajet dans le cas où le balayage est effectué sur la totalité de chaque surface de mesure.

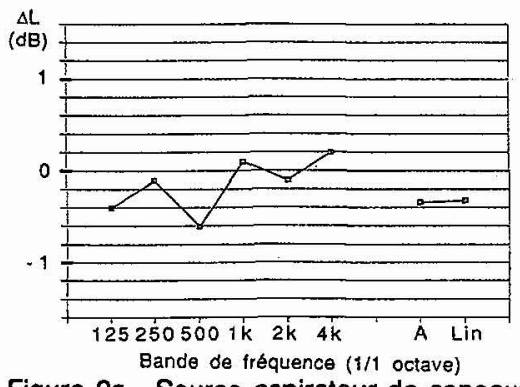

Figure 2a - Source aspirateur de copeaux en chambre semi-anéchoïque.

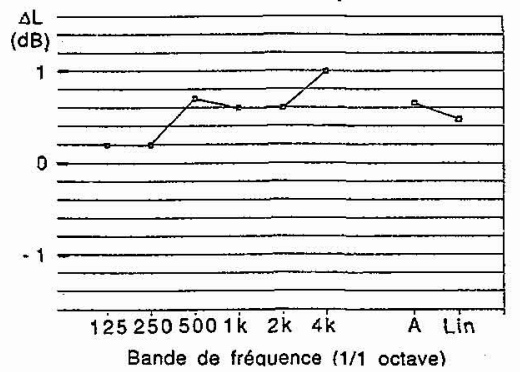

Figure 2c - Climatiseur sur site.

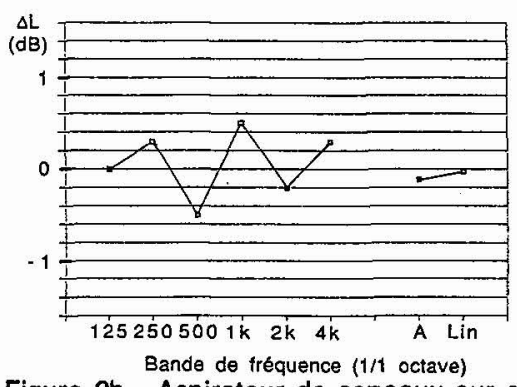

Figure $2 b$ - Aspirateur de copeaux sur site.

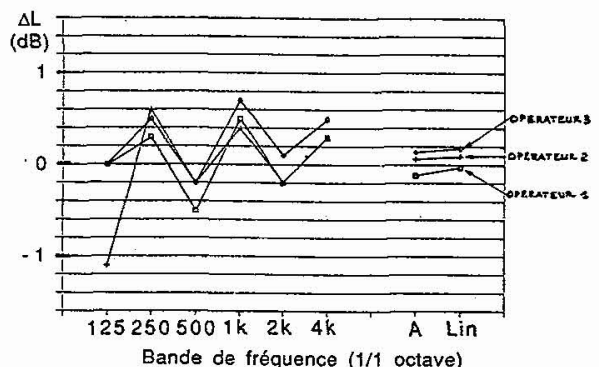

Figure 2d - Aspirateur de copeaux sur site, avec 3 opérateurs.

Figure 2 - Ecart de puissance acoustique $\Delta L$ entre les résultats obtenus par la technique de balayage $L_{w}$ (bal) et ceux obtenus par la technique des points fixes (Pts fixes) $\Delta L=L_{w}$ (bal) $-L_{w}$ (Pts fixes). 